\title{
Welfare and Pricing of Mail in a Communications Market $^{1}$
}

\author{
Philippe De Donder ${ }^{2}$, Helmuth Cremer $^{3}$, \\ Paul Dudley ${ }^{4}$ and Frank Rodriguez ${ }^{5}$
}

April 2, 2010

\footnotetext{
${ }^{1}$ This paper has been presented at the sixth conference on "Regulation, competition and universal service in the postal sector", held in Toulouse on March 25-26, 2010. The analysis contained in this paper reflects the views of the authors and may not necessarily be those of Royal Mail Group.

${ }^{2}$ Toulouse School of Economics (IDEI and GREMAQ-CNRS)

${ }^{3}$ Toulouse School of Economics (IDEI, GREMAQ and Institut universitaire de France)

${ }^{4}$ Royal Mail Group.

${ }^{5}$ Oxera.
} 


\begin{abstract}
We build a model where a postal incumbent offering single piece, transactional and advertising mail competes with postal entrants and with a firm offering an alternative medium. We solve for the optimal prices under various competition assumptions. We calibrate the model and provide numerical simulations in order to shed light on the impact of these assumptions on volumes and welfare levels.

Keywords: (non) direct mail, substitution, displacement ratio, Ramsey prices, calibration.
\end{abstract}




\section{Introduction}

Since there is a minimum mailing level for senders in the bulk mail market, this mail is particularly attractive to the universal service provider (USP) and competing postal entrants, in comparison to individual and smaller mailings of singlepiece mail. The traditional bulk mail market can be thought of as serving business communication needs with other businesses and customers, and includes transactional and advertising mail. Through transactional mail the sender is able to meet its obligation of providing information to the recipient (examples include bank statements and utilities' invoices). Through advertising mail the sender is able to provide information to the recipient to encourage a response or purchase. While the bulk mail market might also comprise of mail used for other purposes, these two form the focus of this paper.

In recent years mail volumes have been in decline within single piece and bulk mail. While this is in part a consequence of the economic downturn, it is also due to the substitution of mail to other communication media. Single piece mail has been substituted by email; transactional mail has been substituted by statements and invoices conveyed by email; and advertising mail has been substituted by alternative digital communication available, for example, through the internet (Soteri et al, 2009).

Optimal pricing within a global price cap and pricing with workshare discounts in the bulk mail market have been explored in the literature (Billette de Villemeur et al 2002, 2003). The effects on welfare and pricing of access and bypass mail competition in a mails market comprising of a single piece and bulk mail services have also been looked at in previous literature (De Donder et al 2006, 2008). Further, the effects on welfare and pricing of a downturn in mail volumes and potential changes to service quality have also been considered (De Donder et al 2010).

As a further development of this earlier literature, this paper looks at welfare and pricing where the bulk mail market is considered to comprise of two distinct markets, of transactional and advertising mail, which differ both in their demand price elasticities (but not in their costs for the postal operators providing them) and in the availability of an alternative medium for advertising but not for transactional mail. We first develop an analytical model with the objective of studying the second-best optimal prices as functions of which goods are available in the economy. In this part of the paper, we obtain variations of well-known optimality

formulas, adapted to the specificities of the postal sector and of the two variants of bulk mail. The main value added by the paper to the literature rests in the numer- 
ical simulations of a calibrated version of this analytical model. These simulations allow us to compare equilibrium levels of variables, such as prices, volumes, but also consumer surplus (welfare) and contributions of various goods to the USP breaking even. As such the paper provides some further understanding of public interest and pricing policy issues for the mails market within a communication market whose relevance to and influence on the mails market has been increasing in recent years.

\section{The model}

There are three markets, one single-piece and two markets in bulk mail (BM): direct mail (advertising mail, denoted by DM) and non direct mail (transactional mail, denoted by NDM). For simplicity, we assume that demands in the three markets are independent from each other (this is not crucial but simplifies the calibration and numerical solving of the model without affecting qualitatively the results). Throughout the paper, the universal service provider (USP) has a monopoly, whether de jure or de facto, over single piece mail, and may face competition in the two bulk mail markets. For simplicity, we assume a competitive fringe of identical entrants on both bulk mail markets.

On the NDM (transactional) market, the competitors are access-based postal operators. They offer a good which is an imperfect substitute to the NDM product sold by the USP. On the DM (advertising) market, competitors are both (digital) media firms not using any postal network, and access-based postal operators. The three goods offered on this market (the two postal goods and the alternative medium) are imperfect substitutes.

To summarize, the USP offers three end-to-end products (single-piece mail, DM and NDM) and two access products (one geared to DM postal operators, the other to NDM postal operators).

We now introduce some notation. Subscripts stand for the operator: $I$ for the incumbent postal operator, $E$ for an entrant postal firm and $A$ for alternative medium firm. The superscript denotes the product: $x$ for single-piece mail, $y$ for non direct mail and $z$ for direct mail or alternative medium.

Let $x$ denote the quantity of single-piece mail and $p^{x}$ its price.

We denote by $y_{I}$ the quantity of NDM sold by the USP, and by $p_{I}^{y}$ its price. Similarly, we denote by $y_{E}$ the quantity of NDM sold by the postal entrants, and by $p_{E}^{y}$ its price. For each unit of entrant's NDM delivered by the USP on behalf of entrants, entrants pay an access charge of $a^{y}$. 
Let $z_{I}$ denote the quantity of DM sold by the USP, and $p_{I}^{z}$ its price. We denote by $z_{E}$ the quantity of DM sold by the entrants, and by $p_{E}^{z}$ its price. We denote by $z_{A}$ the quantity of non postal good (alternative medium) competing with the entrants' and USP's DM and its unit price by $p_{A}^{z}$. For each unit of entrant's DM delivered by the USP, entrants pay an access charge of $a^{z}$.

As for costs, we anticipate on the calibration elements presented in section 4 in order to simplify the notation. The cost structure which seems the most adapted to the USP in the postal sector (see De Donder et al. 2006, 2008, 2010 and the references therein) is that of a fixed cost, which we denote by $F$, coupled to (constant) marginal upstream and downstream costs. ${ }^{1}$ We assume that $c^{x}$ is the (constant unit) collection cost for single-piece, while $d^{x}$ is the (constant unit) single-piece mail's delivery cost. As for the USP, the two categories of BM (DM and NDM) do not differ in either collection or delivery costs: we denote by $c_{I}^{y z}$ the unit and constant collection cost of both DM and NDM for the USP, and by $d_{I}^{y z}$ the unit constant delivery cost of BM (DM and NDM) for the USP. The calibration elements presented in section 4 are such that the USP's unit delivery costs are similar for all three goods, so that we denote $d_{I}=d_{I}^{y z}=d^{x}$.

As for postal entrants, we denote by $c_{E}^{y}$ their collection cost for NDM and by $c_{E}^{z}$ their collection cost for DM. Also, to simplify notation, we assume that $c_{E}^{z}=c_{E}^{y}=c_{E}^{y z}$. As postal entry is through access only (both for DM and NDM), we do not need to introduce an entrant's delivery cost.

Finally, $e_{A}$ is the alternative media operator's constant unit cost of the nonpostal good competing with the USP's and entrants' DM.

We now turn to the demand side. There is one representative sender of mail (and buyer of non postal good competing with the DM bulk mail products), whose utility is

$$
\begin{aligned}
& u(x)+v\left(y_{I}, y_{E}\right)+w\left(z_{I}, z_{E}, z_{A}\right) \\
& -p^{x} x-p_{I}^{y} y_{I}-p_{E}^{y} y_{E}-p_{I}^{z} z_{I}-p_{E}^{z} z_{E}-p_{A}^{z} z_{A}+I,
\end{aligned}
$$

where $I$ is the sender's exogenous income. By maximizing this utility with respect to quantities $\left(x, y_{I}, y_{E}, z_{I}, z_{E}\right.$ and $\left.z_{A}\right)$, we obtain the demand functions

$$
x\left(p^{x}\right), y_{I}\left(p_{I}^{y}, p_{E}^{y}\right), y_{E}\left(p_{I}^{y}, p_{E}^{y}\right), z_{I}\left(p_{I}^{z}, p_{E}^{z}, p_{A}^{z}\right), z_{E}\left(p_{I}^{z}, p_{E}^{z}, p_{A}^{z}\right) \text { and } z_{A}\left(p_{I}^{z}, p_{E}^{z}, p_{A}^{z}\right) .
$$

${ }^{1}$ This means that average costs are decreasing with volume and that the three markets (SP, DM, NDM) are related, even with independent demands, when the USP is subject to a (global) break even constraint. 
The USP's profit function is given by

$$
\begin{aligned}
\Pi_{I}= & \left(p^{x}-c^{x}-d_{I}\right) x\left(p^{x}\right)+\left(p_{I}^{y}-c_{I}^{y z}-d_{I}\right) y_{I}\left(p_{I}^{y}, p_{E}^{y}\right) \\
& +\left(a^{y}-d_{I}\right) y_{E}\left(p_{I}^{y}, p_{E}^{y}\right)+\left(p_{I}^{z}-c_{I}^{y z}-d_{I}\right) z_{I}\left(p_{I}^{z}, p_{E}^{z}, p_{A}^{z}\right) \\
& +\left(a^{z}-d_{I}\right) z_{E}\left(p_{I}^{z}, p_{E}^{z}, p_{A}^{z}\right)-F .
\end{aligned}
$$

Since all entrants (postal and otherwise) behave as a competitive fringe, we have that $p_{E}^{y}=c_{E}^{y z}+a^{y}, p_{E}^{z}=c_{E}^{y z}+a^{z}$ and $p_{A}^{z}=e_{A}$, and that profit is nil for the three kinds of entrants.

The planner's social welfare is given by

$$
\begin{aligned}
& W=u(x)+v\left(y_{I}, y_{E}\right)+w\left(z_{I}, z_{E}, z_{A}\right)-\left(c^{x}+d_{I}\right) x \\
& -\left(c_{I}^{y z}+d_{I}\right)\left(z_{I}+y_{I}\right)-\left(c_{E}^{y z}+d_{I}\right) y_{E}-\left(c_{E}^{y z}+d_{I}\right) z_{E}-e_{A} z_{A}+I .
\end{aligned}
$$

We now turn to the scenarios we study in this paper. They mainly differ in the set of products that are available in the market. For each scenario, we study the optimal second-best (Ramsey) prices.

\section{Scenarios studied}

\subsection{Monopoly}

In this first scenario, we assume that the USP has a monopoly on the three markets, and we compare allocations with and without uniform pricing for the two types of bulk mail. Utility, demand and profit functions are given by a simplified version of the functions above where we set the entrants' quantities to zero to obtain utility functions $v^{M}\left(y_{I}\right), w^{M}\left(z_{I}\right)$ (the superscript $M$ stands for monopoly) and demand functions $y_{I}^{M}\left(p_{I}^{y}\right), z_{I}^{M}\left(p_{I}^{z}\right)$ together with

$$
\begin{aligned}
& \Pi_{I}^{M}=\left(p^{x}-c^{x}-d_{I}\right) x\left(p^{x}\right)+\left(p_{I}^{z}-c_{I}^{y z}-d_{I}\right) z_{I}^{M}\left(p_{I}^{z}\right) \\
& +\left(p_{I}^{y}-c_{I}^{y z}-d_{I}\right) y_{I}^{M}\left(p_{I}^{y}\right)-F,
\end{aligned}
$$

and

$$
\begin{aligned}
& W^{M}=u(x)+v^{M}\left(y_{I}^{M}\right)+w^{M}\left(z_{I}^{M}\right)-\left(c^{x}+d_{I}\right) x \\
& -\left(c_{I}^{y z}+d_{I}\right)\left(z_{I}^{M}+y_{I}^{M}\right)+I .
\end{aligned}
$$

As in De Donder et al. (2006, 2008), we assume that the USP maximizes welfare under the constraint that it breaks even. These Ramsey prices can also be 
attained by subjecting the USP to an optimal global price cap (see Laffont and Tirole, 2000). For pedagogical reasons, we start with the case of differentiated prices, where Ramsey prices solve

$$
\max _{p^{x}, p_{I}^{y}, p_{I}^{z}} W^{M} \text { such that } \Pi_{I}^{M} \geq 0
$$

We obtain the following well known first-order conditions

$$
\begin{aligned}
\frac{p^{x}-c^{x}-d_{I}}{p^{x}} & =\frac{\lambda}{1+\lambda} \frac{1}{\varepsilon^{x}}, \\
\frac{p_{I}^{y}-c_{I}^{y z}-d_{I}}{p_{I}^{y}} & =\frac{\lambda}{1+\lambda} \frac{1}{\varepsilon^{y}}, \\
\frac{p_{I}^{z}-c_{I}^{y z}-d_{I}}{p_{I}^{z}} & =\frac{\lambda}{1+\lambda} \frac{1}{\varepsilon^{z}},
\end{aligned}
$$

where $\lambda$ is the Lagrange multiplier of the non-negative profit constraint, and where

$$
\begin{aligned}
\varepsilon^{x} & =-p^{x} \frac{\partial x\left(p^{x}\right) / \partial p^{x}}{x\left(p^{x}\right)}, \\
\varepsilon^{y} & =-p_{I}^{y} \frac{\partial y_{I}^{M}\left(p_{I}^{y}\right) / \partial p_{I}^{y}}{y_{I}^{M}\left(p_{I}^{y}\right)}, \\
\varepsilon^{z} & =-p_{I}^{z} \frac{\partial z_{I}^{M}\left(p_{I}^{z}\right) / \partial p_{I}^{z}}{z_{I}^{M}\left(p_{I}^{z}\right)},
\end{aligned}
$$

are, respectively, the direct price elasticities of the demand for single-piece mail, non direct mail $y$ and direct mail $z$ when the USP has a monopoly over these three products.

Observe from these equations that the optimal, second best Ramsey prices call for differentiated bulk mail prices as soon as demand price elasticities differ between DM and NDM, even in our environment where their costs are identical. Observe also that an alternative pricing scheme, such as the equiproportional markup, would lead to the same price for the two types of bulk mail, since the same markup would be posted on the same costs for the two goods.

We now study the case where, because for instance of regulatory constraints, the same price is posted for both the DM and NDM. We then solve the following program

$$
\max _{p^{x}, p_{I}^{y z}} W^{M} \text { such that } \Pi_{I}^{M} \geq 0 \text { and } p_{I}^{y}=p_{I}^{z}=p_{I}^{y z}
$$


to obtain the following first-order conditions

$$
\begin{aligned}
\frac{p^{x}-c^{x}-d_{I}}{p^{x}} & =\frac{\lambda}{1+\lambda} \frac{1}{\varepsilon^{x}}, \\
\frac{p_{I}^{y z}-c_{I}^{y z}-d_{I}}{p_{I}^{y z}} & =\frac{\lambda}{1+\lambda} \frac{1}{\varepsilon^{y z}},
\end{aligned}
$$

where

$$
\varepsilon^{y z}=-p_{I}^{y z} \frac{\partial\left[y_{I}^{M}\left(p_{I}^{y z}\right)+z_{I}^{M}\left(p_{I}^{y z}\right)\right] / \partial p_{I}^{y z}}{y_{I}^{M}\left(p_{I}^{y z}\right)+z_{I}^{M}\left(p_{I}^{y z}\right)}
$$

is the demand price elasticity for the whole of bulk mail, and can be expressed as

$$
\varepsilon^{y z}=\frac{y_{I}^{M}\left(p_{I}^{y z}\right) \varepsilon^{y}+z_{I}^{M}\left(p_{I}^{y z}\right) \varepsilon^{z}}{y_{I}^{M}\left(p_{I}^{y z}\right)+z_{I}^{M}\left(p_{I}^{y z}\right)},
$$

i.e., as a weighted average of the demand price elasticities for DM and NDM, with the weights being the market share of these two products in the bulk mail market.

Observe first that the formula for the optimal single-piece price is the same whether bulk mail prices are differentiated or not. This is due to our assumption that demand for single-piece mail and bulk mail are independent. This does not mean that the optimal price levels will be the same, because the value of the Lagrange multiplier of the budget constraint, $\lambda$, will typically differ.

Second, the optimal uniform bulk mail price is given by the usual inverse elasticity rule, where the relevant elasticity pertains to the whole bulk mail market. Note that, because the value of $\lambda$ typically differs between the two problems (1) and (5), we can not state that the optimal uniform bulk mail price (6) is some average of the optimal differentiated DM (4) and NDM (3) prices.

Since adding a constraint always (weakly) decreases the objective, we can be sure that the welfare level attained under (5) is lower than that attained under (1). To quantify this loss of welfare, we have to calibrate the model, which we do in section 4. Observe that, because of the global break even constraint including an overall fixed cost, consumers of single-piece mail are also affected by the introduction of a uniform pricing constraint on the bulk mail market (even though demands are independent across markets). The numerical simulations performed in section 5 will allow us to quantify this effect and to compare it with the impact on the consumers of bulk mail. 


\subsection{Introduction of alternative medium}

In this section, we assume that the USP retains its monopoly position on both the single-piece and NDM market, but faces competition from an alternative medium in the DM market.

In that case, we have utility functions $v^{M}\left(y_{I}\right), w^{P M}\left(z_{I}, z_{A}\right)$ (obtained by setting $z_{E}=0$ in function $w\left(z_{I}, z_{E}, z_{A}\right)$, and where the superscript $P M$ stands for "postal monopoly") and demand functions $y_{I}^{M}\left(p_{I}^{y}\right), z_{I}^{P M}\left(p_{I}^{z}, p_{A}^{z}\right), z_{A}^{P M}\left(p_{I}^{z}, p_{A}^{z}\right)$, together with

$$
\begin{aligned}
\Pi_{I}^{P M}= & \left(p^{x}-c^{x}-d_{I}\right) x\left(p^{x}\right)+\left(p_{I}^{y}-c_{I}^{y z}-d_{I}\right) y_{I}^{M}\left(p_{I}^{y}\right) \\
& +\left(p_{I}^{z}-c_{I}^{y z}-d_{I}\right) z_{I}^{P M}\left(p_{I}^{z}, p_{A}^{z}\right)-F,
\end{aligned}
$$

and

$$
\begin{aligned}
W^{P M}= & u(x)+v^{M}\left(y_{I}^{M}\right)+w^{P M}\left(z_{I}^{P M}, z_{A}^{P M}\right)-\left(c^{x}+d_{I}\right) x \\
& -\left(c_{I}^{y z}+d_{I}\right)\left(y_{I}^{P M}+z_{I}^{P M}\right)-e_{A} z_{A}^{P M}+I .
\end{aligned}
$$

Ramsey prices solve

$$
\max _{p^{x}, p_{I}^{z}, p_{I}^{y}} W^{P M} \text { such that } \Pi_{I}^{P M} \geq 0
$$

which gives the following first-order conditions

$$
\begin{aligned}
\frac{p^{x}-c^{x}-d_{I}}{p^{x}} & =\frac{\lambda}{1+\lambda} \frac{1}{\varepsilon^{x}}, \\
\frac{p_{I}^{y}-c_{I}^{y z}-d_{I}}{p_{I}^{y}} & =\frac{\lambda}{1+\lambda} \frac{1}{\varepsilon^{y}}, \\
\frac{p_{I}^{z}-c_{I}^{y z}-d_{I}}{p_{I}^{z}} & =\frac{\lambda}{1+\lambda} \frac{1}{\varepsilon_{P M}^{z}},
\end{aligned}
$$

where

$$
\varepsilon_{P M}^{z}=-p_{I}^{z} \frac{\partial z_{I}^{P M}\left(p_{I}^{z}, p_{A}^{z}\right) / \partial p_{I}^{z}}{z_{I}^{P M}\left(p_{I}^{z}, p_{A}^{z}\right)} .
$$

The only difference with the optimal monopoly prices (2) to (4) is in the computation of the relevant direct price elasticity for the direct mail market, which has now to be done taking into account the existence of the imperfect substitute represented by the alternative medium. Observe that the cross-price elasticity of the alternative medium demand with respect to the USP's direct mail price 
does not appear in the formula because of our assumption that the alternative media firms behave like a competitive fringe. With an alternative medium's price equal to marginal cost, variations in the quantity of alternative medium when $p_{I}^{z}$ is increased only have second order effects on total welfare. This being said, the optimal price levels will differ from (2) to (4) since the value of $\lambda$ will typically differ.

Here also, we need numerical simulations to illustrate the impact of this scenario on prices, volumes and welfare.

\subsection{Competition on both BM markets}

We now build on the previous section and we introduce access-based (postal) competition on both bulk mail markets (DM and NDM). In that case, utility, demand, profit and welfare functions correspond to those developed in section 2 .

Ramsey prices solve

$$
\max _{p^{x}, p_{I}^{z}, p_{I}^{y}, a^{y}, a^{z}} W \text { such that } \Pi_{I} \geq 0
$$

with the following first-order conditions:

$$
\begin{array}{ll}
\text { for } p^{x}: & (1+\lambda)\left(p^{x}-c^{x}-d_{I}\right) \frac{\partial x}{\partial p^{x}}+\lambda x=0, \\
\text { for } p_{I}^{y}: & (1+\lambda)\left[\left(p_{I}^{y}-c_{I}^{y z}-d_{I}\right) \frac{\partial y_{I}}{\partial p_{I}^{y}}+\left(a^{y}-d_{I}\right) \frac{\partial y_{E}}{\partial p_{I}^{y}}\right]+\lambda y_{I}=0, \\
\text { for } a^{y}: & (1+\lambda)\left[\left(p_{I}^{y}-c_{I}^{y z}-d_{I}\right) \frac{\partial y_{I}}{\partial p_{E}^{y}}+\left(a^{y}-d_{I}\right) \frac{\partial y_{E}}{\partial p_{E}^{y}}\right]+\lambda y_{E}=0, \\
\text { for } p_{I}^{z}: & (1+\lambda)\left[\left(p_{I}^{z}-c_{I}^{y z}-d_{I}\right) \frac{\partial z_{I}}{\partial p_{I}^{y}}+\left(a^{y}-d_{I}\right) \frac{\partial z_{E}}{\partial p_{I}^{z}}\right]+\lambda z_{I}=0, \\
\text { for } a^{z}: & (1+\lambda)\left[\left(p_{I}^{z}-c_{I}^{y z}-d_{I}\right) \frac{\partial z_{I}}{\partial p_{E}^{z}}+\left(a^{z}-d_{I}\right) \frac{\partial y_{E}}{\partial p_{E}^{z}}\right]+\lambda z_{E}=0 .
\end{array}
$$

Observe the symmetry between the first-order conditions for DM (good $z$ ) and NDM (good $y$ ): the availability of the alternative medium as an imperfect substitute to direct mail plays no role in the above formulas. This is due to two assumptions: i) marginal cost pricing by alternative media firms and ii) no access is provided to these firms -i.e., the USP does not make money on the selling of alternative medium. 
To understand these FOCs, observe that increasing one USP price (say, $p_{I}^{y}$ ) affects both the USP's E2E quantity $\left(y_{I}\right)$ but also the quantity of the imperfect substitute offered by the postal entrants $\left(y_{E}\right)$. Each variation in quantity is multiplied by the margin made by the USP on this product and then weighted by one plus the Lagrange multiplier of the USP profit constraint. Increasing the E2E price has also a direct effect on revenue which is measured by the USP's quantity $\left(y_{I}\right)$, which receives a weight of $\lambda$ since it increases profit.

We can now reformulate these conditions in terms of elasticities:

$$
\begin{aligned}
p^{x} & =c^{x}+d_{I}+\frac{\lambda}{1+\lambda} \frac{p^{x}}{\varepsilon^{x}}, \\
p_{I}^{y} & =c_{I}^{y z}+d_{I}+\frac{\lambda}{1+\lambda} \frac{p_{I}^{y}}{\varepsilon_{I}^{y}}+\left(a^{y}-d_{I}\right) \sigma_{E I}^{y}, \\
a^{y} & =d_{I}+\frac{\lambda}{1+\lambda} \frac{a^{y}}{\varepsilon_{E}^{y}}+\left(p_{I}^{y}-c_{I}^{y z}-d_{I}\right) \sigma_{I E}^{y}, \\
p_{I}^{z} & =c_{I}^{y z}+d_{I}+\frac{\lambda}{1+\lambda} \frac{p_{I}^{z}}{\varepsilon_{I}^{z}}+\left(a^{z}-d_{I}\right) \sigma_{E I}^{z}, \\
a^{z} & =d_{I}+\frac{\lambda}{1+\lambda} \frac{a^{z}}{\varepsilon_{E}^{z}}+\left(p_{I}^{z}-c_{I}^{y z}-d_{I}\right) \sigma_{I E}^{z},
\end{aligned}
$$

where

$$
\begin{aligned}
\varepsilon^{x} & =-p^{x} \frac{\partial x\left(p^{x}\right) / \partial p^{x}}{x\left(p^{x}\right)}, \\
\varepsilon_{I}^{y} & =-p_{I}^{y} \frac{\partial y_{I}\left(p_{I}^{y}, p_{E}^{y}\right) / \partial p_{I}^{y}}{y_{I}\left(p_{I}^{y}, p_{E}^{y}\right)}, \\
\varepsilon_{E}^{y} & =-a^{y} \frac{\partial y_{E}\left(p_{I}^{y}, p_{E}^{y}\right) / \partial p_{E}^{y}}{y_{I}\left(p_{I}^{y}, p_{E}^{y}\right)}, \\
\varepsilon_{I}^{z} & =-p_{I}^{z} \frac{\partial z_{I}\left(p_{I}^{z}, p_{E}^{z}, p_{A}^{z}\right) / \partial p_{I}^{z}}{z_{I}\left(p_{I}^{z}, p_{E}^{z}, p_{A}^{z}\right)} \\
\varepsilon_{E}^{z} & =-a^{z} \frac{\partial z_{E}\left(p_{I}^{z}, p_{E}^{z}, p_{A}^{z}\right) / \partial p_{E}^{z}}{z_{E}\left(p_{I}^{z}, p_{E}^{z}, p_{A}^{z}\right)},
\end{aligned}
$$


are the direct price elasticities of demands and

$$
\begin{aligned}
\sigma_{E I}^{y} & =-\frac{\partial y_{E}\left(p_{I}^{y}, p_{E}^{y}\right) / \partial p_{I}^{y}}{\partial y_{I}\left(p_{I}^{y}, p_{E}^{y}\right) / \partial p_{I}^{y}}, \\
\sigma_{I E}^{y} & =-\frac{\partial y_{I}\left(p_{I}^{y}, p_{E}^{y}\right) / \partial p_{E}^{y}}{\partial y_{E}\left(p_{I}^{y}, p_{E}^{y}\right) / \partial p_{E}^{y}}, \\
\sigma_{E I}^{z} & =-\frac{\partial z_{E}\left(p_{I}^{z}, p_{E}^{z}, p_{A}^{z}\right) / \partial p_{I}^{z}}{\partial z_{I}\left(p_{I}^{z}, p_{E}^{z}, p_{A}^{z}\right) / \partial p_{I}^{z}}, \\
\sigma_{I E}^{z} & =-\frac{\partial z_{I}\left(p_{I}^{z}, p_{E}^{z}, p_{A}^{z}\right) / \partial p_{E}^{z}}{\partial z_{E}\left(p_{I}^{z}, p_{E}^{z}, p_{A}^{z}\right) / \partial p_{E}^{z}},
\end{aligned}
$$

are displacement ratios, in the spirit of Armstrong (2008) and De Donder (2006).

Optimal prices are then given by the sum of three terms: marginal costs, the inverse elasticity term and a third term. This last term takes into account the fact that increasing one USP price displaces demand from that good to the postal substitute. This displacement effect is measured by the displacement ratio, and is weighted by the margin made selling this good (either directly in the case of an end-to-end product, or through access). Displacement to the alternative medium does not appear in the above formulas because i) this good is offered at marginal cost and ii) the provision of this good does not bring revenues to the USP, as no access is offered for that product.

We could also impose the constraint that the access charge has to be the same for both bulk mail products. The formulas we would obtain would be more complex, without bringing much new intuition (the optimal uniform access charge would be some weighted average of the determinants of the optimal differentiated access charges for DM and NDM).

We resort to calibrations and numerical simulation in order to shed light on how optimal levels (as opposed to formulas) are affected by these different scenarios.

\section{Calibration}

Our calibration assumptions are based on De Donder et al $(2006,2008)$ modified to take account of the fact that bulk mail is split between direct mail and nondirect mail. The assumptions are not estimates from a particular postal operator, but our assessment is that they reflect well the general nature of postal markets and cost structures given published empirical studies.

We start from the hypothetical situation where the USP does not face postal or non-postal competition (see section 3.1 above). We assume that the USP sets a 
price of $0.50 €$ for the single piece product and a price of $0.40 €$ for DM and NDM. Total quantities sold at those prices are $2 \mathrm{bn}, 2 \mathrm{bn}$ and $6 \mathrm{bn}$ items, respectively. The direct price elasticities are -0.2 for single piece, -1.0 for $\mathrm{DM}$ and -0.2 for NDM (consistent with a price elasticity for bulk mail of -0.4 used in previous papers (De Donder et al 2006, 2008). ${ }^{2}$ We calibrate linear demands based on these quantities, prices and elasticities.

We need further information to calibrate the demand functions for DM and NDM products when first an alternative medium competes with DM and second postal entrants compete with the USP's DM and NDM products through downstream access to the USP's network. We use two types of information: the extent of entry for different price configurations and the substitutability between the products within the DM-Advertising market (which by the end includes the products of the USP, postal entrant and alternative medium) and the NDM market (which by the end includes the products of the USP and postal entrant). With regards to the extent of entry, we assume for NDM that the entrant's market share is $10 \%$ if its price is the same as the USP's and $50 \%$ if $20 \%$ cheaper. $^{3}$ For DM, we first have to define the measurement unit for alternative medium. We assume that one "item" of alternative medium refers to the quantity of this medium that is necessary to have the response rate as one item of DM. ${ }^{4}$ Equipped with this definition, we assume that the alternative medium's market share is $25 \%$ if $50 \%$ cheaper than the USP and 35\% if $75 \%$ cheaper than the USP and that the alternative medium's market share is $30 \%$ if $50 \%$ cheaper than the entrant and $40 \%$ if $75 \%$ cheaper than the entrant. On substitution, we assume the displacement ratio of mail transferring to the alternative medium, or postal entrant in NDM or DM is 0.75 , which means that three quarters of the quantities sold by entrants are effectively displaced from the USP, while one quarter represents additional volumes sold in the sector. As such, the USP and postal entrant products are assumed to be close substitutes and the alternative medium a more distant substitute to them.

The USP unit upstream cost is equal to $0.18 €$ for single piece and $0.12 €$ for

\footnotetext{
${ }^{2}$ These are closely aligned to observed price elasticities (see Thress (2006) and Veruete-McKay et al (2010)).

${ }^{3}$ We make these assumptions at the calibration prices. With linear demands, the market shares will typically differ for other price configurations where the entrant is $10 \%$ or $20 \%$ cheaper than the USP.

${ }^{4} \mathrm{So}$, if the alternative medium is email, and if the response rate of prospective customers to email is, say, 20 times less than for DM, then one item of alternative medium corresponds to 20 emails.
} 
the DM and NDM products. The USP delivery cost is $0.12 €$ for single piece, DM and NDM items. Hence the end-to-end unit costs are the same for the USP's DM and NDM products. The value of the fixed costs $F$ equals $1.680 \mathrm{bn} €$ so that the USP breaks even in the hypothetical monopoly situation. The alternative medium is assumed to have a cost of $0.20 €$ per item. The postal entrant's upstream cost is set at $0.15 €$ per item. As in previous papers (De Donder et al 2006, 2008), entrants do not face any fixed cost.

These assumptions determine the linear demand functions and costs for the calibrated model consistent with the analytical presentation in this paper (see Annex 1 for further details).

\section{$5 \quad$ Calibrated results}

We set out below the results of the calibrated model, starting with the monopoly case with differentiated DM and NDM prices and moving to the case where these prices are uniform, before considering the cases where an alternative medium is introduced to the DM market and then where, in addition, competitive postal entry through access to the USP's network is introduced for both DM and NDM.

\subsection{Monopoly with differentiated Ramsey prices}

In the first column of figures in Table 1 we show the calibrated monopoly position (where $p_{I}^{x}=0.5 €$ and $p_{I}^{y}=p_{I}^{z}=0.4 €$ ), and in the second column we show the second-best (Ramsey) solution where $p_{I}^{y}$ and $p_{I}^{z}$ can be different (see section 3.1). The second best outcome has a higher price at $0.426 €$ for NDM (the low elasticity BM good) and a lower price at $0.288 €$ for DM (the BM good with a larger elasticity) although their marginal costs are the same- with a weighted average price of $0.384 €$ - and higher single piece price (of $0.532 €$ ). Total volumes increase from $10 \mathrm{bn}$ to $10.457 \mathrm{bn}$ items. While the USP still breaks even, the profit contribution from the DM service is reduced (by 0.197bn€) and is offset by increases from single piece (by 0.058bn€) and the NDM service (by 0.139bn€). Total consumer surplus increases from $8.900 \mathrm{bn} €$ to $8.939 \mathrm{bn} €$, as the reduction in consumer surplus for single piece customers (of $0.063 \mathrm{bn} €$ ) and NDM customers (of $0.152 \mathrm{bn} €$ ) is more than offset by the increase in consumer surplus for DM customers (of $0.255 \mathrm{bn} €$ ). 
Table 1: Calibrated results for the USP monopoly

\begin{tabular}{|c|c|c|c|c|}
\hline & & \multicolumn{3}{|c|}{ Monopoly } \\
\hline \multirow[t]{2}{*}{ Reference } & & \multicolumn{2}{|c|}{ Section 5.1} & \multirow[b]{2}{*}{$\begin{array}{l}\text { Section } 5.2 \\
\text { With same } \\
\text { prices for } \\
\text { NDM and } \\
\text { DM }\end{array}$} \\
\hline & & Calibration & $\begin{array}{l}\begin{array}{l}\text { With } \\
\text { different }\end{array} \\
\text { prices for } \\
\text { NDM and } \\
\text { DM }\end{array}$ & \\
\hline \multicolumn{5}{|l|}{ Prices $(€)$ : } \\
\hline Single piece & $p_{I}^{x}$ & 0.500 & 0.532 & 0.609 \\
\hline NDM - USP & $p_{I}^{y}$ & 0.400 & 0.426 & 0.373 \\
\hline DM - USP & $p_{I}^{Z}$ & 0.400 & 0.288 & 0.373 \\
\hline \multicolumn{5}{|l|}{ Quantities (bn): } \\
\hline Single piece & $x_{I}^{X}$ & 2.000 & 1.974 & 1.913 \\
\hline NDM - USP & $y_{I}$ & 6.000 & 5.932 & 6.082 \\
\hline DM - USP & $Z_{I}$ & 2.000 & 2.560 & 2.137 \\
\hline Total & & 10.000 & 10.457 & 10.133 \\
\hline \multicolumn{5}{|c|}{$\begin{array}{l}\text { Contribution to fixed cost - } \\
\text { USP (€bn): }\end{array}$} \\
\hline Single piece & & 0.400 & 0.458 & 0.590 \\
\hline NDM & & 0.960 & 1.099 & 0.806 \\
\hline DM & & 0.320 & 0.123 & 0.283 \\
\hline Total profit (€bn): & & 0.000 & 0.000 & 0.000 \\
\hline \multicolumn{5}{|c|}{ Consumer surplus (€bn) } \\
\hline Single piece & & 2.500 & 2.437 & 2.288 \\
\hline NDM & & 6.000 & 5.848 & 6.166 \\
\hline DM & & 0.400 & 0.655 & 0.457 \\
\hline Total & & 8.900 & 8.939 & 8.910 \\
\hline Lagrange multiplier & & & 0.104 & 0.148 \\
\hline
\end{tabular}




\subsection{Monopoly with uniform Ramsey bulk mail prices}

When a uniform price constraint is applied to bulk mail (where $p_{I}^{y}=p_{I}^{z}$ ), the second best outcome for the monopoly is shown in the third column of figures in Table 1. The uniform price for bulk mail $(0.373 €)$ is in between the optimal differentiated prices for NDM $(0.426 €)$ and for DM $(0.288 €)$ and lower than the

weighted average of the two from column 2. Conversely, the single-piece price increases from $0.532 €$ to $0.609 €$ so that not differentiating prices for bulk mail by market segment results in a significant rise in the single piece price even though bulk mail and single piece products are not modelled as substitutes. Total volumes reduce from $10.457 \mathrm{bn}$ to $10.133 \mathrm{bn}$ items. The USP still breaks even, with the profit contribution increases for the DM service (by $0.160 \mathrm{bn} €$ ) and single piece (by $0.132 \mathrm{bn} €$ ) offset by a profit reduction from the NDM service. The Lagrange multiplier increases from 0.104 to 0.148 , because the uniform constraint makes it more difficult for the USP to raise money in the postal market, and total consumer surplus reduces from 8.939bn€ to 8.910bn€; reductions in consumer surplus for single piece customers (of $0.149 \mathrm{bn} €$ ) and DM customers (of 0.198bn€) are not offset by the increase in consumer surplus for NDM customers (of $0.318 \mathrm{bn} €$ ). Hence, welfare reduces with the uniform price constraint for bulk mail, and the burden of it is borne by the single piece and DM customers, to the benefit of the NDM customers. The ranking of welfare levels for the various cases in Table 1 follows that obtained from the analytical part of the paper.

\subsection{Competition in DM through an alternative medium}

When competition from an alternative medium is introduced to DM, and the USP can differentiate in price between DM and NDM (see section 3.2), the second best outcome is shown in the first column of figures in Table 2 (which can be compared to the second column of figures in Table 1). The introduction of competition to DM drives the USP price lower in the DM-Advertising market (from $0.288 €$ to $0.274 €)$, and higher in the other two markets although observe that the size of the variations in prices is quite small. The weighted average price for DM and NDM increases marginally (from $0.384 €$ to $0.386 €$ ) and the single piece price also increases (from $0.532 €$ to $0.539 €$ ). Total volume in the joint DM and alternative medium part of the Advertising market increases from 2.56 billion items to 2.697billion items, and the alternative medium corresponds to $11.7 \%$ of this market in volume, with reductions in volumes for both single piece and NDM. The Lagrange multiplier increases only marginally from 0.104 to 0.107 with the 
Table 2: Calibrated results for the USP subject to competition from an alternative medium to DM and competition from entry through access to the USP's network

\begin{tabular}{|c|c|c|c|c|c|c|c|}
\hline \multirow{4}{*}{$\begin{array}{l}\text { Reference } \\
\text { Assumptions }\end{array}$} & & \multicolumn{6}{|c|}{ Competition } \\
\hline & & \multirow{3}{*}{$\begin{array}{l}\begin{array}{l}\text { DM medium } \\
\text { only }\end{array} \\
\text { Section } 5.3 \\
\end{array}$} & \multicolumn{5}{|c|}{ DM medium plus access entry } \\
\hline & & & \multirow{2}{*}{ Section 5.4} & \multicolumn{4}{|c|}{ Section 5.5: Sensitivities } \\
\hline & & & & & & & \\
\hline With USP DM & & Yes & Yes & No & Yes & Yes & Yes \\
\hline Displacement ratio & & 0.75 & 0.75 & 0.75 & $\underline{0.90}$ & 0.75 & 0.75 \\
\hline Price elasticity (DM/NDM) & & $-1.0 /-0.2$ & $-1.0 /-0.2$ & $-1.0 /-0.2$ & $-1.0 /-0.2$ & $-0.8 /-0.267$ & $-1.0 /-0.2$ \\
\hline $\begin{array}{l}\text { Medium share for } 50 \% \\
\text { price reduction }\end{array}$ & & $25 \%$ & $25 \%$ & $25 \%$ & $25 \%$ & $25 \%$ & $\underline{75 \%}$ \\
\hline \multicolumn{8}{|l|}{ Prices $(€)$ : } \\
\hline Single piece & $p_{I}^{x}$ & 0.538 & 0.537 & 0.541 & 0.539 & 0.581 & 0.546 \\
\hline NDM - USP & $p_{I}^{y}$ & 0.430 & 0.430 & 0.433 & 0.431 & 0.413 & 0.437 \\
\hline NDM - access & $a^{y}$ & - & 0.265 & 0.267 & 0.295 & 0.252 & 0.270 \\
\hline NDM - entrants & $p_{E}^{y}$ & - & 0.415 & 0.417 & 0.434 & 0.402 & 0.420 \\
\hline DM - USP & $p_{I}^{z}$ & 0.276 & 0.276 & - & 0.274 & 0.288 & 0.263 \\
\hline DM - medium & $p_{A}^{z}$ & 0.200 & 0.200 & 0.200 & 0.200 & 0.200 & 0.200 \\
\hline DM - access & $a^{z}$ & - & 0.150 & 0.150 & - & 0.160 & 0.138 \\
\hline DM - entrants & $p_{E}^{z}$ & - & 0.300 & 0.300 & - & 0.310 & 0.288 \\
\hline \multicolumn{8}{|l|}{ Quantities (bn): } \\
\hline Single piece & $x_{I}^{x}$ & 1.970 & 1.970 & 1.967 & 1.969 & 1.935 & 1.963 \\
\hline NDM - USP & $y_{I}$ & 5.909 & 5.249 & 5.241 & 5.779 & 5.300 & 5.230 \\
\hline NDM - entrants & $y_{E}$ & - & 0.882 & 0.881 & 0.442 & 0.867 & 0.879 \\
\hline DM - USP & $\mathrm{z}_{I}$ & 2.380 & 2.012 & - & 2.383 & 1.805 & 1.432 \\
\hline DM-medium & $z_{A}$ & 0.317 & 0.295 & 0.446 & 0.272 & 0.305 & 1.515 \\
\hline DM - entrants & $Z_{E}$ & - & 0.493 & 2.114 & - & 0.521 & 0.147 \\
\hline Total & & 10.576 & 10.900 & 10.648 & 10.546 & 10.733 & 11.166 \\
\hline \multicolumn{8}{|l|}{$\begin{array}{l}\text { Contribution to fixed cost } \\
\text { - USP (€bn): }\end{array}$} \\
\hline Single piece & & 0.469 & 0.468 & 0.475 & 0.470 & 0.543 & 0.483 \\
\hline NDM & & 1.125 & 0.997 & 1.011 & 1.103 & 0.915 & 1.030 \\
\hline DM & & 0.087 & 0.073 & - & 0.082 & 0.087 & 0.033 \\
\hline Access NDM & & - & 0.128 & 0.130 & 0.025 & 0.115 & 0.132 \\
\hline Access DM & & & 0.015 & 0.064 & - & 0.021 & 0.003 \\
\hline Total profit (€bn): & & 0.000 & 0.000 & 0.000 & 0.000 & 0.000 & 0.000 \\
\hline & & & & & & & \\
\hline
\end{tabular}




\begin{tabular}{|l|l|l|l|l|l|l|l|}
\hline Consumer surplus (€bn): & & & & & & & \\
\hline Single piece & & 2.425 & 2.426 & 2.418 & 2.423 & 2.341 & 2.409 \\
\hline NDM & & 5.819 & 5.833 & 5.815 & 5.816 & 4.436 & 5.792 \\
\hline $\begin{array}{l}\text { Advertising (DM and } \\
\text { alternative media) }\end{array}$ & & 0.707 & 0.714 & 0.587 & 0.708 & 0.784 & 1.094 \\
\hline Total & & 8.951 & 8.973 & 8.820 & 8.947 & 7.560 & 9.294 \\
\hline & & & & & & & 0.131 \\
\hline Lagrange multiplier & & 0.107 & 0.107 & 0.109 & 0.109 & 0.111 \\
\hline
\end{tabular}


alternative medium making it more difficult for the USP to break even. The USP still breaks even, with the profit contribution decrease for the DM service (by $0.036 \mathrm{bn} €$ ) offset by the profit increase from single piece (by $0.011 \mathrm{bn} €$ ) and the NDM service (by 0.026bn€). The total consumer surplus increases (from $8.939 \mathrm{bn} €$ to $8.951 \mathrm{bn} €$ ) with the added choice of the alternative medium; reductions in consumer surplus for single piece customers (of 0.012bn€) and NDM customers (of $0.029 \mathrm{bn} €$ ) are more than offset by the increase in consumer surplus for DM customers (of 0.052bn€). The increase in consumer surplus for DM is constrained by the displacement ratio of 0.75 making the alternative medium a relatively close substitute for DM and the calibration of the alternative medium having low market shares even when significantly cheaper than DM. Nevertheless, the introduction of the alternative medium enhances welfare and still allows the USP to break even within the calibration used.

\subsection{Competition through an alternative medium in DM and access to the USP's network for NDM and DM}

When competition from postal entry to the USP's downstream network is introduced for NDM and DM along with the alternative medium to DM, and the USP can differentiate in price between DM and NDM (see section 3.3), the second best outcome is shown in the second column of figures in Table 2 and can be compared with its first column of figures. The USP's single piece, NDM and DM prices are the same or little changed. In line with the analytical part of the paper, although the marginal cost of access for both of these products is the same the USP offers differentiated access prices of $0.265 €$ and $0.150 €$ for NDM and DM respectively. This leads to the postal entrant having volume of $0.882 \mathrm{bn}$ and $0.493 \mathrm{bn}$ items for NDM and DM respectively. This is not only acquired from the USP volumes, as the alternative medium volumes also reduce (from $0.315 \mathrm{bn}$ to $0.295 \mathrm{bn}$ items) and there is growth in overall volumes as a result of entry of a differentiated product. The contribution made by single piece, NDM and DM to the USP's break even position is little changed, but the consumer surplus increases for each. Total volumes increase (to 10.900bn items) and total consumer surplus increases (to 8.973bn€). The introduction of entry through access leads to differentiated access prices for DM and NDM and an outcome with both USP break even and enhanced welfare within the calibration used. 


\subsection{Sensitivity analysis}

The final four columns of Table 2 shows, in turn, the effect of a change in one of four assumptions used in the main calibration. These are discussed briefly below with comparison in each case being with the results in the second column of Table 2 .

If the USP does not offer a DM end-to-end service (in the first column of sensitivities - see Annex 2 for an analytical description), the USP raises its prices for all its remaining services other than DM access (which remains at the same price). DM customers transfer volume from the USP to the alternative medium and the postal entrant, but the DM and total market volumes reduce. Consumer surplus reduces in each market and overall. Hence the withdrawal of the DM service by the USP leads to a reduction in welfare for all customers, and particularly so for DM customers. ${ }^{5}$

If the displacement ratio is 0.9 rather than 0.75 (in the second column of sensitivities), it reduces the volume growth arising from the transfer of volumes to access. To recover its costs the USP increases all of its prices, but particularly its NDM and DM access prices. This reduces the competitiveness of postal entry through access and, in this case, it can only compete in the NDM market. Consumer surplus decreases in each market and overall, but the reduction is limited in DM by the USP and postal entrant service being closer substitutes.

If the difference between the own price elasticities for DM and NDM is reduced (such that the price elasticities are -0.8 and -0.267 for DM and NDM respectively, thereby retaining an overall bulk mail elasticity of -0.4 in the calibration), it raises the second best postal prices for DM and single piece and reduces them for NDM (in the third column of sensitivities). The USP gains volumes in NDM and loses volumes in DM, with converse movements for the postal entrant and a marginal increase in volumes for the alternative medium. However, overall, the main effect is a reduction in DM and total market volumes. There is also a higher Lagrange multiplier, reflecting tougher conditions for the USP to break even, which in the welfare-maximising solution it achieves by raising the single piece price substantially. ${ }^{6}$

If the market share for the alternative medium were to be $75 \%$ instead of $25 \%$

\footnotetext{
${ }^{5}$ Recall that this "contagion effect" is due to the imposition of a global break even constraint on the USP, with a global fixed cost to be financed by several markets, even though demands are independent across markets.

${ }^{6}$ Note that the absolute value of consumer surplus changes for DM and NDM due to the effect of the alternative elasticity values on the demand functions for these products.
} 
when its price is $50 \%$ less than the USP's price (in the fourth column of sensitivities), significantly more volumes transfers from DM to the alternative medium and the overall volume of the DM-Advertising market increases significantly. The USP increases its prices for single piece and NDM, and reduces them in DM to break even, and the postal entrant has similar price movements in NDM and DM. The volume growth and price reductions in DM-Advertising increase consumer surplus, and outweigh milder reductions in consumer surplus in single piece and NDM. While there is a mildly higher Lagrange multiplier, reflecting marginally tougher conditions for the USP to break even, which is achieved by raising the single piece price and NDM prices, total consumer welfare increases substantially.

Finally, we also looked at a case where the postal competitors charge was assumed to be a fixed mark up over marginal cost. This assumption does not change qualitatively the results.

\section{Conclusions}

The development of the mails market within a communications market, where there is increased competition from medium outside of the postal sector, has a significant bearing on the optimal pricing and future of the universal service provision. Within this paper we have developed a framework for assessing both, with scope to develop further insight of competition and regulatory impacts.

In setting prices in the bulk mail market, there are good economic grounds for the USP to differentiate its prices between services with different price elasticities (in this case direct mail and non-direct mail services) even when their marginal costs are the same. This is well known to academic economists, but we observe that very few network operators differentiate price purely on the basis of demand because they can rely on cost differences to justify pricing and also in some cases because of the existence of regulatory and legally oriented constraints. We show how welfare improves without a uniform price constraint in such a bulk mail market. Part of this improvement in welfare from differentiating prices in the bulk mail market arises from the possibility this creates for single piece prices to then be lowered and so benefit single piece customers.

The introduction of alternative medium competing with direct mail has the effect of increasing customer choice and welfare. Within the calibrations used here the USP continues to break even with the introduction of an alternative and competing medium, primarily because the effect of that medium on USP volumes and prices is limited. Where these effects are greater and the USP is subject to 
price regulation, the USP might not break even.

The introduction of competition in the bulk mail market, together with the alternative medium, enhances further customer choice and welfare. Observe that this need not be the case, since the introduction of alternative medium drives the USP to increase its prices in other markets in order to break even. Conversely, a withdrawal of the USP from end to end direct mail reduces customer choice and welfare.

While it might appear that it would always be better to have all competing service options available to customers, within our calibrations we show an example where this is not the case. When the welfare gains from the introduction of access services is limited, the second best (Ramsey) outcome has the USP price for access resulting in there being only competition in DM-Advertising from the alternative medium, and not from postal competition.

Further, with greater competition from the alternative medium to direct mail over time, the USP will face greater pressure to break even. Within our calibrations, greater competition from the alternative medium causes the USP to increase prices elsewhere to compensate for the effect of the loss in volumes to the alternative medium. For the second best (Ramsey) outcome, the effect on the USP is likely to be greater, the closer are the own price elasticities for DM and NDM.

\section{Annex 1: Calibration}

Assuming linear demands, the three utility functions are given by

$$
\begin{aligned}
u(x) & =a x-\frac{b}{2} x^{2}, \\
v\left(y_{I}, y_{E}\right) & =c y_{I}-(d / 2) y_{I}^{2}+e y_{E}-(f / 2) * y_{E}^{2}-g y_{I} y_{E}, \\
w\left(z_{I}, z_{E}, z_{A}\right) & =h z_{I}-(i / 2) z_{I}^{2}+j z_{E}+(k / 2) z_{E}^{2}+l z_{A}+(m / 2) z_{A}-n z_{I} z_{E}-o z_{I} z_{A}-p z_{E} z_{A} .
\end{aligned}
$$

We use the calibration assumptions described in section 4 to set the values of the 16 parameters ( $a$ to $p$ ), and to obtain the following demand functions, on the single-piece mail market:

$$
x\left(p^{x}\right)=2.4-0.8 p^{x},
$$

on the NDM market

$$
\begin{aligned}
y_{I}^{M}\left(p_{I}^{y}\right) & =7.2-3 p_{I}^{y} \\
y_{I}\left(p_{I}^{y}, p_{E}^{y}\right) & =4.1011+26.3736 p_{E}^{y}-22.7802 p_{I}^{y}, \\
y_{E}\left(p_{I}^{y}, p_{E}^{y}\right) & =4.13187-35.1648 p_{E}^{y}+26.3736 p_{I}^{y},
\end{aligned}
$$


and on the DM market

$$
\begin{aligned}
z_{I}^{M}(p) & =4 .-5 p_{I}^{z} \\
z_{I}^{P M}\left(p_{I}^{z}, p_{A}^{z}\right) & =3.77534+1.75342 p_{A}^{z}-6.31507 p_{I}^{z} \\
z_{A}^{P M}\left(p_{I}^{z}, p_{A}^{z}\right) & =0.299543-2.3379 p_{A}^{z}+1.75342 p_{I}^{z} \\
z_{I}\left(p_{I}^{z}, p_{E}^{z}, p_{A}^{z}\right) & =2.31752+1.19272 p_{A}^{z}+12.9439 p_{E}^{z}-16.023 p_{I}^{z} \\
z_{E}\left(p_{I}^{z}, p_{E}^{z}, p_{A}^{z}\right) & =1.94376+0.747605 p_{A}^{z}-17.2586 p_{E}^{z}+12.9439 p_{I}^{z} \\
z_{A}\left(p_{I}^{z}, p_{E}^{z}, p_{A}^{z}\right) & =0.215344-2.37028 p_{A}^{z}+0.747605 p_{E}^{z}+1.19272 p_{I}^{z}, \\
z_{E}^{N}\left(p_{E}^{z}, p_{A}^{z}\right) & =3.81593+1.71113 p_{A}^{z}-6.80203 p_{E}^{z}, \\
z_{A}^{N}\left(p_{E}^{z}, p_{A}^{z}\right) & =0.387855-2.2815 p_{A}^{z}+1.71113 p_{E}^{z} .
\end{aligned}
$$

\section{Annex 2: No USP E2E DM product}

We build on section 3.3 and we assume that the USP does not provide an end-to-end DM product, so that the only postal product in the DM market is the access-based mail offered by the entrant, which competes with the alternative medium product. Such a situation could arise for instance if the alternative medium were priced well below the USP direct mail marginal cost $\left(e_{A}<c_{I}^{y z}+d_{I}\right)$ so that the demand for the USP end-to-end direct mail product is driven to zero.

The analysis for single-piece mail and non direct mail (good $y$ ) are the same as in section 3.3, leading to the same optimality formulas for the prices (of course, optimal price levels will typically differ). We then concentrate on the direct mail market $(\operatorname{good} z)$. For this market, we have the utility function $w^{N}\left(z_{E}, z_{A}\right)$ (where the superscript $N$ stands for "no E2E USP DM product") obtained by setting $z_{I}=0$ in $w\left(z_{I}, z_{E}, z_{A}\right)$ and demand functions $z_{E}^{N}\left(p_{E}^{z}, p_{A}^{z}\right)$ and $z_{A}^{N}\left(p_{E}^{z}, p_{A}^{z}\right)$, together with

$$
\begin{aligned}
\Pi_{I}^{N}= & \left(p^{x}-c^{x}-d_{I}\right) x\left(p^{x}\right)+\left(p_{I}^{y}-c_{I}^{y z}-d_{I}\right) y_{I}\left(p_{I}^{y}, p_{E}^{y}\right) \\
& +\left(a^{y}-d_{I}\right) y_{E}\left(p_{I}^{y}, p_{E}^{y}\right) \\
& +\left(a^{z}-d_{I}\right) z_{E}^{N}\left(p_{E}^{z}, p_{A}^{z}\right)-F
\end{aligned}
$$

and

$$
\begin{aligned}
& W^{N}=u(x)+v\left(y_{I}, y_{E}\right)+w^{N}\left(z_{E}^{N}, z_{A}^{N}\right)-\left(c^{x}+d_{I}\right) x \\
& -\left(c_{I}^{y z}+d_{I}\right) y_{I}-\left(c_{E}^{y z}+d_{I}\right) y_{E}-\left(c_{E}^{y z}+d_{I}\right) z_{E}^{N}-e_{A} z_{A}^{N}+I .
\end{aligned}
$$

Ramsey prices solve

$$
\max _{p^{x}, p_{I}^{y}, a^{y}, a^{z}} W^{N} \text { such that } \Pi_{I}^{N} \geq 0 .
$$


The optimal access price on the direct mail market is given by the inverse elasticity rule

$$
a^{z}=d_{I}+\frac{\lambda}{1+\lambda} \frac{a^{z}}{\varepsilon_{E}^{z N}}
$$

where

$$
\varepsilon_{E}^{z N}=-a^{z} \frac{\partial z_{E}^{N}\left(p_{E}^{z}, p_{A}^{z}\right) / \partial p_{E}^{z}}{z_{E}^{N}\left(p_{E}^{z}, p_{A}^{z}\right)}
$$

\section{References}

[1] Armstrong, M. (2008) "Access Pricing, Bypass and Universal Service in Post", Review of Network Economics, 7: 172-187.

[2] Billette de Villemeur, E, H. Cremer, B. Roy and J. Toledano (2002), "Access and (Non)-Uniform Pricing in the Postal Sector", in: Competitive transformation of the postal and delivery sector, edited by M. A. Crew and P. R. Kleindorfer, Boston: Kluwer Academic Publishers, 43-68.

[3] Billette de Villemeur, E, H. Cremer, B. Roy and J. Toledano (2003), "Pricing and worksharing discounts in the postal sector", in: Postal and Delivery Services: Delivering on Competition, edited by M. A. Crew and P. R. Kleindorfer, Boston: Kluwer Academic Publishers, 33-48.

[4] De Donder Ph. (2006), "Access Pricing in the Postal Sector: Theory and Simulations", Review of Industrial Organization, 28-3: 307-326

[5] De Donder Ph., Cremer H., Dudley P. and Rodriguez F. (2006), "Pricing and Welfare Implications of Alternative Approaches to Setting Price Controls in the Postal Sector", in: Progress toward Liberalization of the Postal and Delivery Sector, edited by M.A. Crew and P.R. Kleindorfer, Springer, 227248.

[6] De Donder Ph., Cremer H., Dudley P. and Rodriguez F. (2008), "Pricing, Welfare and Organisational Constraints for Postal Operators", in: Competition and Regulation in the Postal and Delivery Sector, edited by M.A. Crew and P.R. Kleindorfer, Cheltenham: Edward Edgar,150-166.

[7] De Donder Ph., Cremer H., Dudley P. and Rodriguez F. (2010), "Welfare and Profit Implications for Changes in Service Specifications within the Universal 
Service", in: Heightening Competition in the Postal and Delivery Sector, edited by M.A. Crew and P.R. Kleindorfer, Cheltenham: Edward Edgar, 161-180.

[8] Laffont, J.J. and J. Tirole (2000), Competition in Telecommunications. Cambridge, MA: MIT Press.

[9] Soteri, S., F. Feve, J-P. Florens and F. Rodriguez (2009), "Internet advertising and direct mail: trends and analysis for the UK", in Progress in the Competition Agenda in the Postal and Delivery Sector, edited by M.A. Crew and P.R. Kleindorfer, Cheltenham: Edward Edgar, 209-222.

[10] Thress, T. (2006), Direct Testimony of Thomas Thress on Behalf of the United States Postal Service, docket R2006-1, Postal Regulatory Commission, Washington, DC.

[11] Veruete-McKay, L., S. Soteri, J. Nankervis and F. Rodriguez (2011), "Letter traffic demand in the UK: an analysis by product and envelope content type", Review of Network Economics, forthcoming. 